Rhode Island College

Digital Commons @ RIC

Master's Theses, Dissertations, Graduate

Master's Theses, Dissertations, Graduate

Research and Major Papers Overview

Research and Major Papers

2016

\title{
Effects of Hyperglycemia on Adults Hospitalized With Sepsis
}

Katie Cardoso
Rhode Island College

Follow this and additional works at: https://digitalcommons.ric.edu/etd

Part of the Other Nursing Commons

\section{Recommended Citation}

Cardoso, Katie, "Effects of Hyperglycemia on Adults Hospitalized With Sepsis" (2016). Master's Theses, Dissertations, Graduate Research and Major Papers Overview. 142.

https://digitalcommons.ric.edu/etd/142

This Major Paper is brought to you for free and open access by the Master's Theses, Dissertations, Graduate Research and Major Papers at Digital Commons @ RIC. It has been accepted for inclusion in Master's Theses, Dissertations, Graduate Research and Major Papers Overview by an authorized administrator of Digital Commons @ RIC. For more information, please contact digitalcommons@ric.edu. 


\section{EFFECTS OF HYPERGLYCEMIA ON ADULTS HOSPITALIZED \\ WITH SEPSIS}

by

\section{Katie Cardoso}

A Major Paper Submitted in Partial Fulfillment of the Requirements for the Degree of

Master of Science in Nursing

in

The School of Nursing

Rhode Island College

2016 


\begin{abstract}
Infectious sepsis (IS) is the result of a complex infection that is difficult to identify, diagnosis and treat. The gold standard for treatment of IS is early identification and intervention. There is a rise in patients with IS along with a concurrent rise of adults with Diabetes Mellitus (DM). Adults with DM are more susceptible to acute illness due to the multiple body systems affected by DM. Acute illness in adults with DM leads to increased blood glucose levels that become difficult to treat. Extreme elevations of blood glucose are linked to longer lengths of stay and higher mortality rates in adults with IS. Surviving Sepsis Campaign recommends blood glucose levels to be controlled below $180 \mathrm{mg} / \mathrm{dl}$ for all adults with and without diabetes. The purpose of this research study was to identify if a relationship exists between hyperglycemia, hyperlactatemia, hospital length of stay and mortality rates in septic adults with diabetes (Group A) and without diabetes (Group B). Findings of the study did not project what was predicted regarding hyperglycemia, however high lactate levels were associated with longer lengths of stay and higher mortality rates as predicted. This retrospective, two group design study found adults without diabetes had longer lengths of stay and higher mortality rates than adults with diabetes indicating poorly controlled glucose levels in nondiabetic adults with sepsis may impact patient outcomes. APRN's can be instrumental in implementing change in patient care and hospital policy by providing evidenced based practice by following practice standards established at a national level such as the Surviving Sepsis Campaign.
\end{abstract}




\section{Table of Contents}

Background/Statement of the Problem ...........................................................................

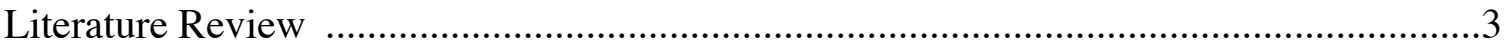

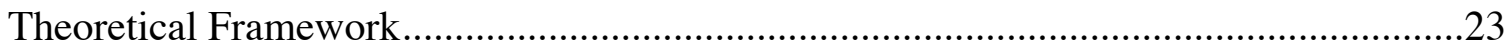

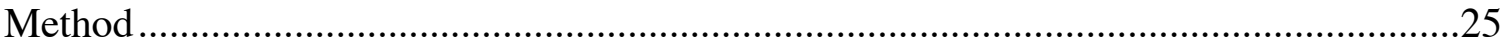

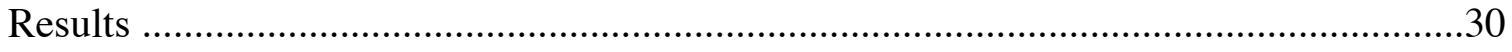

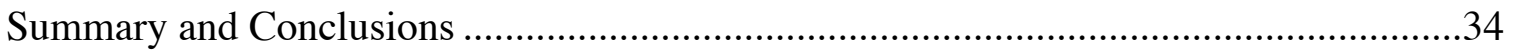

Recommendations and Implications for Advanced Nursing Practice ............................37

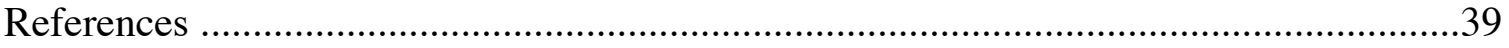

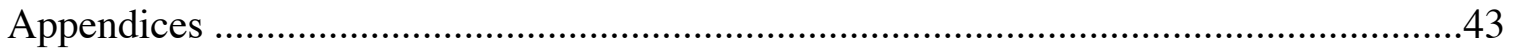




\section{EFFECTS OF HYPERGLYCEMIA ON ADMITTED ADULTS WITH SEPSIS}

\section{Background/Statement of the Problem}

Infectious sepsis (IS) is the result of a complex infection that is not only difficult to predict and diagnose, but to treat as well (Center for Disease Control [CDC], 2015). Early identification and intervention is critical for adults presenting to the emergency department with bacteremia. Adult's with IS manifest infection with systemic signs of inflammation and vital organ dysfunction, and remain at greater risk of death than those suffering from uncomplicated infections. Septic adults are particularly prone to hyperglycemia and insulin resistance due to of a number of pathophysiologic changes associated with the infectious response. Higher rates of IS are causing a rise in national mortality rates. In order to prevent death and provide better outcomes, it is vital to examine the role glucose in hospitalized adults diagnosed with IS (Mayo Clinic, 2014).

Every year more than one million adults in America will become septic, of which $28-50 \%$ will die. The incidence is higher than prostate cancer, breast cancer and HIVAIDS combined. Sepsis accounts for greater than 500,000 emergency department visits, 750,000 hospitalizations, and 215,000 deaths per year in the United States. The number of sepsis cases in the United States has been on the rise, likely due to a combination of factors including an increased sepsis awareness, increased tracking of sepsis, an aging population, increased longevity of people living with chronic disease, the spread of antibiotic-resistant organisms, an upsurge in invasive procedures and the broad use of immunosuppressive and chemotherapeutic agents (CDC, 2015).

In addition to the rising numbers of adults with sepsis, there is also a concurrent rise in the number of adults living with diabetes mellitus (DM). Currently over 29 million people live with DM in the United States, and one out of four are unaware they have the disease. Due to better treatment, people with diabetes are living longer than before. DM affects multiple body systems making adults more susceptible to many chronic and acute illnesses including sepsis. Acute illness in adults with DM leads to 
increased sick days and elevations in blood glucose levels that are prolonged making the disease difficult to control. The risk of death is $50 \%$ higher for adults with DM than those without DM. Approximately $\$ 245$ billion are spent annually on treatment of DM (Mayo Clinic, 2014). It is important to explore whether medical providers should consider hyperglycemia when creating a plan of care for a diabetic versus a non-diabetic adults diagnosed with IS. Little is known about the impact hyperglycemia has on sepsis and the effects on the severity of illness.

Extreme elevations of blood glucose are associated with higher mortality rates in various groups of hospitalized adults. The use of continuous insulin infusions, known as intensive insulin therapy (IIT), improves outcomes in a selected group of critically ill patients with the aim of normoglycemic control (Ali et al., 2008). The current recommendation from the Surviving Sepsis Campaign (2008), includes insulin therapy to maintain glucose below $180 \mathrm{mg} / \mathrm{dL}$ in all septic patients. Glucose variability, rather than the glucose level, has also been shown to be a significant factor associated with inhospital mortality (Ali et al., 2008). Treatment and prevention of hyperglycemia has been advocated for subjects with sepsis (Surviving Sepsis Campaign, 2008). 


\section{Literature Review}

A literature review was conducted utilizing the search engines of CINAHL and Pub Med databases. Key words searched and combined included sepsis, diabetes mellitus, hyperglycemia, stress hyperglycemia, intensive insulin, and general adaptation theory. Literature from 2000 to 2015 was searched. Key literature found in the search will be discussed.

\section{Introduction}

It was once thought that acute hyperglycemia was a normal and beneficial response for critical illness by providing additional glucose to organs in need of increased energy during illness. Hyperglycemia during illness is now being recognized as a harmful response for some adults (Schuetz, Castro \& Shapiro 2011; Kaufman, 2008). It is common to have stress-induced hyperglycemia for both diabetic and non-diabetic adults during critical illness, but the level of which elevated glucose becomes harmful is still being studied. Adults with diabetes are at greater risk for not only developing infection, but becoming septic as well. The risk increases from two and half to six times as likely for a diabetic adult to become septic than a non-diabetic adult (Stegenga, Vincent, Vail, Xie, Hanley, Williams, Bernard, \& Poll, 2010). DM has the potential to alter the inflammatory and coagulation pathways and this makes DM an important comorbid condition to consider in the management of acute sepsis (Schuetz, Castro \& Shapiro, 2011; Stegenga et al., 2010)

\section{Sepsis}

Sepsis, secondary to infection, is a potentially life-threatening event. When sepsis occurs, chemicals are released into the bloodstream to trigger an inflammatory response throughout the body. The inflammatory response triggers a cascade of changes in multiple organ systems and results in organ failure. Once these changes occur, septic shock develops causing low blood pressure, decreased vital organ perfusion which can lead to death. The patient must exhibit at least two of the following symptoms to be 
diagnosed with sepsis: a core body temperature greater than $101^{\circ} \mathrm{F}$ or less than $96.8^{\circ} \mathrm{F}$, heart rate greater than 90 beats per minute, respiratory rate greater than 20 breathes per minute, and probable or confirmed infection. Severe sepsis may be present if one of the following symptoms are present: significantly decreased urine output, abrupt change in mental status, decrease in platelet count, difficulty in breathing, abnormal heart pumping function and/or abdominal pain (Mayo Clinic, 2014). As part of the surviving sepsis campaign, 24 hour management bundle includes: blood product administration, immunoglobulin use, mechanical ventilation in acute respiratory distress syndrome, sedation or analgesia, glucose control, renal replacement therapy, bicarbonate therapy, deep venous thrombosis and stress ulcer prophylaxis, nutrition, and goal setting care. Surviving Sepsis Campaign (SSC) 2015, an international committee of expert's consensus on the guidelines for management of severe sepsis and sepsis shock recommends to initiate insulin after two consecutive blood glucose levels greater than $180 \mathrm{mg} / \mathrm{dl}$, with an upper limit set at $180 \mathrm{mg} / \mathrm{dl}$. Blood glucose values are to be monitored every one to two hours until below $180 \mathrm{mg} / \mathrm{dl}$, then monitored every four hours thereafter. Glucose levels obtained by point-of-care capillary blood are to be used with caution as values may not accurately reflect arterial blood or plasma blood values (Surviving Sepsis Campaign, 2015).

IS has been an increasingly critical medical condition in the last decade, with an increased number of deaths occurring secondary to severe IS. IS accounts for more than 500,000 emergency room visits, 751,000 hospitalizations, and 215,000 deaths per year in the United States (CDC, 2015; Ali et al., 2008; Michaels \& Dorman, 2004). A main goal for optimal sepsis management includes early recognition and treatment aimed at disrupting the pathway. The pathophysiology of sepsis and treatment guidelines are well established, therefore treatment should be standardized, including glucose control (Michaels \& Dorman, 2004). 
A review, conducted by Michaels \& Dorman (2004), supported the need to apply evidence-based guidelines in the ICU to improve the outcomes of IS. The research was based on a retrospective chart review of 26 adults in medical and surgical ICU in an urban community hospitals from January 2003 to October 2003. Michaels \& Dorman identified seven aspects that improved IS outcomes: early goal directed therapy, appropriate and timely administration of antibiotics, availability of blood culture results, source control, appropriate assessment and use of drotrecogin alfa ( a drug class with anti-thrombotic, anti-inflammatory and profibrinolytic properties), appropriate assessment and treatment of adrenal insufficiency, and intensive glucose control. Michaels \& Dorman found many elements of IS guidelines were not implemented during their review period. The mean percentage of adults with sepsis that received evidence based therapies were as follows: appropriate administration of broad-spectrum antibiotics $48.9 \%$, timely administration of broad-spectrum antibiotics $44.2 \%$, appropriate assessment of adrenal insufficiency $22.6 \%$, appropriate use of steroids $31.2 \%$, appropriate assessment for drotrecogin alfa $25.4 \%$, appropriate use of drotrecogin alfa $73.5 \%$, availability of blood culture results $49.1 \%$, appropriate use of vancomycin $50.0 \%$, discontinuation of vancomycin within 96 hours $40.0 \%$, and implementation of tight glucose control 19.3\% (Michaels \& Dorman, 2004). The researchers proposed that by using the guidelines, the increasing mortality rate of sepsis can be largely reduced. Although a small sample, this study demonstrates the wide variability of guideline implementation in this small sample of ICU patients.

Lin et al., 2009 conducted a retrospective study examining 621 bacteremic adults in the emergency department at a university hospital from January 2007 through December 2007. The study was conducted to describe bacteremic adults presenting to emergency departments and identify risk factors for mortality. The data collection included age, sex, underlying illness, clinical condition, microbiology, source of bacteremia, and patient outcomes obtained from electronic medical records. The most 
common underlying illness identified was DM (39.3\%). Mortality rates were as follows: three day mortality $4.0 \%$, seven day mortality $6.8 \%, 14$ day mortality $10.3 \%, 21$ day mortality $11.4 \%$, and 28 day mortality $12.6 \%$. Most frequently isolated pathogen was Escherichia coli (39.2\%), and the most common source of bacteremia was urinary tract infection $(41.2 \%)$ followed by primary bacteremia (13.2\%). The overall 28-day mortality rate was $12.6 \%$. Independent predicting factors for 28-day mortality in bacteremic adults included: age, malignancy, liver cirrhosis, alcohol use, polymicrobial bacteremia, anemia, and sepsis. Lin et al. noted the importance of identifying the risk factors that contribute to mortality in bacteremic adults within the ED setting. Limitations of the study include inconsistencies among completeness of data in medical records and additional risk factors not identified or explored (Lin et al., 2009).

\section{Diabetes Mellitus}

In 2012, the incidence of a new diagnosis of DM was 1.7 million with a total of 29.1 million Americans already living with DM accounting for 9.3\% of the US population. DM remains the seventh leading cause of death in America and a total of 234,051 death certificates listed DM as an underlying or contributing cause of death in 2010. DM is underreported as a cause or contributing factor of death, only $35 \%-40 \%$ of people with diabetes had diabetes listed anywhere on the death certificate. After adjusting for population age and sex differences, average medical expenditures among people diagnosed with DM were 2.3 times higher than without DM (American Diabetes Association, 2015).

The pathophysiology of DM is complex due to the different etiologies causing the similar symptoms, signs and complications. The disease can be classified by etiology and pathology as Type 1 Diabetes Mellitus (T1DM), Type 2 Diabetes Mellitus (T2DM), "other", and gestational diabetes mellitus (GDM). The category of "other" consists of

many problems but accounts for less than $1 \%$ of people with diabetes. GDM affects $5 \%$ to $6 \%$ of pregnant women and in most instances is an early form of T2DM. T1DM 
consists of $5 \%$ to $10 \%$ of people with DM, and T2DM accounts for the remainder of the population, approximately $90 \%$. In all cases of DM, the underlying denominator is the deficiency of the hormone insulin. Although T1DM is commonly known as a genetic disorder, new research is finding multiple environmental contributors to the development of the disease, including coxsackie viruses. Research is still ongoing to discover other environmental precipitating factors. The believed etiology of the pathophysiology of T2DM is still evolving daily with ongoing research. T2DM is not a single disease but a group of diseases or syndromes with different genetic, environmental and pathophysiology factors that have similar symptoms and outcomes. Simplified, T2DM is a combination of insulin resistance and insulin deficiency. Genetic factors contributing to T2DM are still being identified, but environmental factors are well known including age, sedentary lifestyle, and obesity (Guthrie \& Guthrie, 2004).

Proper care of critically ill adults with DM will greatly reduce morbidity and mortality. The use of IV insulin, proper attention to fluid and electrolyte management, frequent neurological exams, and careful physical, electronic, and laboratory monitoring is necessary for ideal outcomes. Adults with elevated glucose levels will often be profoundly dehydrated and very insulin sensitive. Controlled administration of fluids and control of blood glucose levels are mandatory to obtain optimal care of critically ill adults (Guthrie \& Guthrie, 2004).

Hospitalized adults with T2DM may benefit from tight glycemic control to prevent hyperglycemia and complications from high glucose levels. Johnston \& Horn (2011) conducted a retrospective study to compare two groups of inpatients with diabetes receiving subcutaneous insulin via a basal-bolus approach or sliding scale. The study was conducted at a single medical facility in North Carolina, a total of 45 charts were reviewed with the following inclusion criteria: admission to the hospital within the prior year, history of T2DM, length of stay greater than four days, inpatient insulin administration, oral diabetic medication, and septicemia without mechanical ventilation. 
Exclusion criteria were: admission for surgical procedure, inpatient prescriptions of steroids, thiazide diuretics or niacin, receipt of enteral feeding or total parenteral nutrition; diagnosis of renal failure, liver failure, chronic obstructive pulmonary disease, diabetic ketoacidosis, or pancreatitis, admission to intensive care unit, or inpatient prescription of intravenous insulin. Data collected included age, weight, height, sex, race, date of admission, length of stay, discharge diagnostic related groups, inpatient oral diabetic medications, and insulin prescriptions, blood glucose values, and home diabetic medications. Four blood glucose results were collected for each subject daily for three consecutive days. Results from the study indicated $49 \%$ of adults received basal plus correction insulin, and $51 \%$ received correction insulin only. Out of 540 capillary blood glucose $(\mathrm{CBG})$ results collected in total, $56 \%$ were classified as hyperglycemic events and $1 \%$ hypoglycemic events. In the basal plus correction insulin group $70 \%$ were considered hyperglycemic, while in the correction only insulin group $42 \%$ were considered hyperglycemic. Johnston \& Horn concluded that greater than $50 \%$ of the blood glucose levels collected were considered hyperglycemic events indicating optimal glucose control a continuous problem throughout the day and not just an isolated event. These findings support the need for a change in practice in order to improve the glucose levels while adults are hospitalized. Study limitations include a small sample size, use of oral glycemic medications impacted CBG results, varying levels of diabetes, and additional unmeasured variables that could affect blood glucose levels such as preexisting co-morbidities and physiologic stress during hospitalization (Johnston \& Horn, 2011).

\section{Hyperglycemia Stress Response}

Hyperglycemia is defined as having a fasting blood glucose level greater than 106 in healthy adults and greater than 110 in critically ill adults (Thompson, 2008). The effects and long term impact of hyperglycemia on non-diabetic adults is less understood than diabetic adults. Specifically, it is unclear if the association of hyperglycemia and 
mortality in non-diabetic adult is related to the toxic effects of hyperglycemia or if the hyperglycemia is an indication of stress and can be related to severity of illness (Schuetz, Castro, \& Shapiro, 2011). Historically, hyperglycemia has been viewed as a normal response to stress in critically ill adults. Treatment often is not initiated unless blood glucose levels exceed 200 to $250 \mathrm{mg} / \mathrm{dl}$. The specific mechanisms by which hyperglycemia contributes to poor outcomes are not yet known. However, research demonstrates that intensive insulin therapy targeting euglycemia reduces morbidity and mortality in critically ill adults (Digman, Borto, \& Nasraway, 2005).

Schuetz, et al. (2011) conducted a systematic review of 15 studies examining the evidence from preclinical findings and clinical trials to determine whether DM has an impact on sepsis pathophysiology, susceptibility and or clinical outcomes. The researchers found a relationship between hyperglycemia and sepsis in diabetic and nondiabetic adults. They also concluded that insulin provides more than one protective effect. Insulin not only lowers glucose levels, it has a direct effect on cell function and the physiologic response to infection. The reviewers were unable to conclude if adults with DM have worse outcomes then non-diabetic adults. Schuetz et al. purposed future studies focusing on diabetes as a syndrome, considering important confounding factors such as hyperglycemia, obesity, secondary micro- and macrovascular complications, insulin therapy, endothelial dysfunction to better understand the complexity DM and sepsis has in adults. No limitations were identified in the study.

Schuetz, Jones, Howell, Trzeciak, Ngo, Younger, Aird, \& Shapiro (2011) conducted a prospective study with three independent cohorts from two large tertiary care centers. The study included a total of 7,754 adults admitted to the hospital from the emergency department with suspected infections. The participants were observed for an association between diabetes and mortality rates. The study adjusted for age, sex, disease severity and sepsis syndrome. Results indicated a $4.3 \%$ morality rate $95 \%$ CI [3.9\% to $4.8 \%$ ], with similar findings in diabetic and nondiabetic adults, $4.1 \%$ versus $4.4 \%$ 
absolute risk difference $(0.4 \% ; 95 \%$ CI $[0.7 \%$ to $1.4 \%])$. There was no significant association between DM and mortality in adjusted analysis (odds ratio [OR] 0.85; 95\% CI [0.71 to 1.01$])$. However, diabetes significantly modified the effect of hyperglycemia and hypoglycemia with mortality; initial glucose levels higher than $200 \mathrm{mg} / \mathrm{dl}$ were associated with higher mortality in nondiabetic patients (OR 2.1; 95\% CI [1.4 to 3.0]) but not in diabetic patients (OR 1.0; 95\% CI 0.2 to 4.7), whereas glucose levels less than 100 $\mathrm{mg} / \mathrm{dl}$ were associated with higher mortality in diabetic adults (OR 2.3; 95\% CI 1.6 to 3.3 ) and to a lesser extent in nondiabetic adults (OR 1.1; 95\% CI 1.03 to 1.14). Although no significant association was found between diabetes and mortality rates within the adjusted analysis, the researchers reported an increased mortality rate for non-diabetic adults with elevated initial blood glucose levels. Limitations to study included adults prospectively enrolled according to suspicion of infection at ED evaluation, thus the study did not integrate longitudinal information and some adults ultimately found to have a noninfectious diagnosis may have falsely been included. The study did not include type and duration of diabetes or weight of adult, which could provide further insight (Schuetz et al., 2011).

\section{Hyperlactatemia}

A marker for metabolic stress response is hyperlactatemia. The severity and duration of hyperlactatemia are related to mortality in critically ill adults. Increased lactate production can occur from tissue hypoxia, or as a response to inflammatory mediators, catecholamines, and other factors affecting sodium potassium and adenosine triphosphatase activity. Conditions such as severe sepsis can be indicated by hyperlactatemia (Revelly, Tappy, Martinez, Bollmann, Cayeux, Berger, \& Chiolero, 2005).

A retrospective chart review was conducted by Green, et al. (2012) from 2007 through 2009 at an urban teaching hospital. A total of 1, 236 ED adults were included to determine if an association exists between hyperglycemia, hyperlactatemia and mortality 
rates. Study subjects were identified from emergency room encounters. The data was collected from the electronic medical records (EMR). Nondiabetic adults, suspected to have an infection, with two or more systemic inflammatory response syndrome (SIRS) criteria, and simultaneous lactate and glucose testing in the ED, were included in the review. Two logistic regression models were created to determine if coexisting hyperlactatemia affects hyperglycemia's association with mortality. A total of $9.3 \%$ of adults were hyperglycemic, $13 \%$ were hyperlactatemic, and $17 \%$ of adults died. The researchers concluded septic nondiabetic adults did not have higher mortality rates due to hyperglycemia unless associated with coexisting hyperlactatemia (95\% confidence interval, $(\mathrm{OR}=3.96,95 \% \mathrm{CI}$ [2.01 to 7.79$])$. Limitations of the study include selection bias as patients were previously identified and categorized, the data collected may have led to misclassification. Due to retrospective data collection, illness severity score was not able to be used. A further limitation is that adults were not followed prospectively after discharge to account for mortality rates after illness (Green, et al. 2012).

A prospective observational study by Revelly, et al. (2005), was conducted to evaluate the relative importance of increased lactate production as opposed to decreased lactate utilization in hyperlactatemic adults in the relation to glucose metabolism. The study took place in an ICU of a university hospital and consisted of seven adults with severe sepsis, seven adults with cardiogenic shock, and seven healthy volunteers. Interventions consisted of infusing sodium lactate at $10 \mathrm{umol} / \mathrm{kg} / \mathrm{min}$ and then 20 umol $/ \mathrm{kg} / \mathrm{min}$ over 120 minutes each to all study subjects, for a total of 2 infusions. Glucose was infused throughout the duration of the infusions of lactate. Baseline arterial lactate levels, heart rate, blood pressure, and cardiac index were all obtained prior to infusion and every 60 minutes post infusion, to observe glucose turnover and lactate conversion to glucose, for a total of 12 hours. Plasma glucose and insulin concentrations were monitored. Oxygen consumption and $\mathrm{CO} 2$ production were measured before and after infusions. The researchers found that baseline arterial lactate was higher in patients 
with severe sepsis $(3.2 \pm 2.6)$ or cardiogenic shock $(2.8 \pm 0.4)$ than in healthy volunteers $(0.9 \pm 0.20 \mathrm{mmol} / \mathrm{L}, \mathrm{p}<0.5)$. Lactate clearance was similar in all three groups: severe septic $10.8 \pm 5.4$, cardiogenic shock $9.6 \pm 2.1$, and healthy volunteers $12.0 \pm 2.6$ $\mathrm{ml} / \mathrm{kg} / \mathrm{min}$. Endogenous lactate production, determined as the initial lactate concentration multiplied by lactate clearance, was markedly enhanced in septic adults $(26.2 \pm 10.5)$ and cardiogenic adults $(26.6 \pm 5.1)$ compared to healthy volunteers $(11.2 \pm 2.7 \mathrm{umol} / \mathrm{kg} / \mathrm{min}$, $\mathrm{p}<0.1)$. Conclusively both, septic and cardiogenic adults had increased lactate production concomitantly to hyperglycemia, and increased glucose turnover, indicating that hyperglycemia substantially influences lactate metabolism during critical illness. Limitations to the study were small sample size and pharmacokinetic evaluation of lactate metabolism relies on a study of basal lactate concentrations.

Lactate testing in septic adults is now a key component to the Surviving Sepsis Campaign (SSC). The National Quality Forum (NQF) ratified the measures for the treatment and management of adults with severe sepsis and septic shock as submitted collaboratively by the Henry Ford Hospital, the Infectious Diseases Society of America, and the Society of Critical Care Medicine. Measures ratified by NQF are considered by the Centers for Medicare and Medicaid (CMS) for public reporting and payment programs. The NQF's adoption of the SSC guidelines means that lactate measurements in severe sepsis could be included in quality measures for public reporting and reimbursements.

Rhee, Murphy, Platt \& Klompas (2015) conducted a retrospective cohort study to analyze lactate testing in suspected septic adults over a span of 11 years. The purpose of the study was to measure the trends of lactate testing in septic patients. The study took place in two U.S. academic hospitals and included adult septic patients admitted from 2003 to 2013 . The annual rates of lactate measurement were assessed in severe septic adults who had blood cultures ordered. Results of the study found rates of lactate measurement in hospitalized adults with blood culture orders increased from $11 \%$ in 2003 
to $48 \%$ in 2013 ( $p<0.001$ ). Rates of repeat lactate measurement within 6 hours after lactate levels greater than or equal to $4.0 \mathrm{mmol} / \mathrm{L}$ increased from $23 \%$ to $69 \%$ (p < $0.001)$. In $2003,49 \%$ of adults were on vasopressors (intravenous blood pressure therapy) at the time of first lactate measurements versus $21 \%$ in $2013(\mathrm{p}<0.001)$. Despite these trends, in 2013 only $65 \%$ of adults with severe sepsis had lactates measured at the time of suspected sepsis. On multivariate analysis, hospital-onset sepsis and hospitalization on a nonmedical service were common predictors for failure to measure lactates (adjusted odds ratio, 7.56; 95\% CI [6.31-9.06] and adjusted odds ratio, 2.08; 95\% CI [1.76-2.24], respectively). The researchers found hospital-onset sepsis and nonmedical units are still suboptimal in measuring serial lactate levels in septic adults despite SSC guidelines (Rhee, Murphy, Platt \& Klompas, 2015).

\section{Risk for Development of Type II Diabetes}

A prospective study by Gornik, Vujaklija, Lukic, Madzarac \& Gasparovic, (2010) was conducted on 744 septic adults, of which 584 were without preadmission diagnosis of diabetes. The purpose of the study was to identify adults at risk for developing T2DM post hyperglycemia during sepsis encounter. It is common for septic adults to develop hyperglycemia as it is a result of the inflammatory response to stress and severity of illness, however not all severely ill adults will develop hyperglycemia. Those in the study who had hyperglycemia and were non-diabetic were found to be at four time's greater risk of developing T2DM five years after illness. Of the 584 adults without diabetes preadmission 55 developed diabetes within the five year follow-up. The relative risk for developing T2DM was 4.29 (95\% CI [1.35 - 13.64]). The researchers found incidences of hyperglycemia to be a risk factor for developing T2DM and proposed that adults within this high-risk population should be followed up closely for the development of diabetes. Limitations include size of study group and follow-up group. Larger study group with longer follow-up is recommended for further research (Gornik et al., 2010).

\section{Intensive Insulin Therapy}


A recommendation from Surviving Sepsis Campaign recommends blood glucose of less the $150 \mathrm{mg} / \mathrm{dL}$. One way of achieving normoglycemia is with intensive insulin therapy (IIT). Intensive insulin therapy is a term used to describe tight management of blood sugar levels. The regimen to manage diabetes in this way includes frequent testing and either regular administration of insulin or use of an insulin pump (American Diabetes Association, 2015). Most critically ill adults benefit greatly from the use of IIT. However, researchers report similar findings of high risk for hypoglycemia with little to no difference in mortality rates compared to adults with moderate hyperglycemia, defined as glucose level between 140mg/dL and 180mg/dL (Surviving Sepsis Campaign, 2015).

Thompson (2008) conducted a systematic review of intensive insulin therapy in septic adults. The review included 60 individual studies which focused on key points including: stress hyperglycemia, IIT, early treatment of glucose control, (renal protective) mechanisms, hypoglycemia, variability of protocols, assessing glucose control and amount of time for nurses to manage glucose control. Stress-induced hyperglycemia was found to be associated with an increase in the risk of death, congestive heart failure, cardiogenic shock after myocardial infarction and an increase in mortality after ischemic stroke. Hyperglycemia was observed to be an independent predictor of poor outcomes, the cause-effect relationship still remains unclear. The authors observed a significantly improved outcome when stress hyperglycemia was controlled within 48 hours of critical illness. Also noted by researcher as a study limitation, glucose values that were tested by point of care capillary blood glucose may overestimate arterial blood glucose when values are low and the degree of overestimation can impact the clinical treatment. According to the authors, it remains unclear if the hypoglycemia experienced from IIT is related to short term mortality. The study proposes guidelines which include the use of a validated insulin titration protocol, glucose monitoring every one to two hours, and the provision of a caloric source while on insulin therapy (Thompson, 2008). 
A prospective study by Lorencio et al, 2012 assessed the accuracy of a real-time continuous glucose monitoring system within an ICU to determine if the status of the septic adult was influenced by the accuracy of the adult's blood glucose. The researchers monitored 41 adults over the course of 72 hours. Adults included in the study were admitted to the ICU with glucose levels greater than $150 \mathrm{mg} / \mathrm{dl}$. The insulin protocol established by the ICU of the hospital was used to obtain glycemic control for the study subjects. Arterial blood samples were taken every 30 minutes until a stable level was reached and then taken every one, two, three or four hours thereafter for monitoring. Adults under the age of 18, pregnant, or who had participated in another study, were excluded in the study. The researchers propose that adults with septic shock had impaired interstitial glucose, therefore improper glucose control can occur without the use of realtime continuous glucose monitoring (RTCGM) directly from the blood stream. Study limitations included small sample size, degree of edema or tissue perfusion was not taken into account, and placement of RTCGM. The authors concluded from the research findings that in septic adults, RTCGM was most accurate at $74.5 \%(\mathrm{P}<0.05)$ when compared to arterial blood glucose samples (Lorencio et al, 2012).

A study by Van Den Berghe (2003) compared normalization of glucose for diabetic and non-diabetic adults in a prospective observational study. The purpose of the study was to determine if hyperglycemia predisposes adults to common ICU complications and was not a beneficial adaptation to severe illness. The study compared 1,548 ICU ventilated septic adults within two university hospitals, $14.7 \%$ of the adults had the pre-diagnosis of diabetes. A randomly assigned intensive insulin therapy group received insulin infusion to control blood glucose levels between $80-110 \mathrm{mg} / \mathrm{dl}$. The conventional treatment group received insulin only in the event that blood glucose levels exceeded $200 \mathrm{mg} / \mathrm{dl}$. Results from the study showed the intensive insulin therapy group had a $43 \%(p=0.036)$, reduction in intensive care mortality, a $34 \%(\mathrm{p}=0.005)$, reduction in hospital mortality reduced risk of severe infection by $46 \%(\mathrm{P}=0.003)$, and a $35 \%(\mathrm{p}<$ 
0.001) reduction in prolonged (greater than 10 days) antibiotic use. Limitations of the study included that the population was limited to ICU intubated septic adults only and was not applied to medical ICU, children ICU or surgical adults on medical wards (Van den Berghe, 2003).

\section{Glucose Variability}

Spapen (2008) compared glycemic variable in adults with sepsis. The study was a retrospective review of data from two landmark studies, examining 950 adults admitted to the ICU with sepsis. Adults were divided into two groups, one group consisting of 471 adults received conventional sliding scale insulin and the other group included 479 adults who received IIT. Hyperglycemia was found to be a common finding in septic adults. Factors that increased the risk for hyperglycemia were presence of insulin resistance, high circulating levels of stress hormones and therapies that promoted hyperglycemia such as steroids, continuous enteral nutrition feedings, and dextrose infusions. The study results indicated that septic adults had a significant increase in hypoglycemic events $(19.6 \%)$ compared to non-septic adults $(6.8 \%)$ when receiving IIT. The researcher observed glucose variability measured by the Glycemic Lability Index as best tool for predicting hospital mortality. The Glycemic Lability Index is defined as the squared difference between consecutive glucose measures per unit of actual time between those samples. Glucose variability, as identified by the index, was associated with increased hospital mortality independent from relevant clinical factors such as hypoglycemia, degree of organ failure, intensity of glucose testing, and treatment. No limitations were identified in the study (Spapen, 2008).

Ali et al. (2008) conducted a study to determine if septic adults are more prone to hyperglycemia and insulin resistance due to the number of pathophysiologic changes caused by the release of catecholamines, cortisol and glucagon associated with sepsis. The purpose of the study was to assess the association of glucose variability and hospital mortality in septic adults. The study was a retrospective chart review from a single-center 
cohort at a tertiary care hospital with no sepsis interventions. The study included 1,246 adults hospitalized for more than one day with a diagnosis of sepsis. Excluded from the study were hospital readmissions, prisoners, and adults with limited glucose data. Results of the study showed glycemic lability index had the best discrimination for mortality $(\mathrm{AUC}=0.67, \mathrm{p}<0.001)$. After adjustments for confounders such as organ failure and hypoglycemic events, Ali et al, found a significant interaction between glycemic lability index, average glucose level, and the odds of hospital mortality. Adults with increased glycemic lability index and lower average glucose values had almost a five-fold increased odds of hospital mortality ( $\mathrm{OR}=4.73,95 \%$ [CI 2.6 to 8.7$])$. The researchers concluded glucose variability to be an independent factor in mortality rates and a need for future research to support the findings of glucose variability to be a new "gold-standard" in adults diagnosed with sepsis. Limitations are as follows: unable to determine timing of organ failure with respect to onset of sepsis, glucose variability, or other unmeasured factors. The study did not use a physiology-based severity of illness score for riskadjustment and missing information about other treatments adults may have received (Ali et al., 2008).

\section{Diabetes and Sepsis}

Diabetes has a high prevalence and a potential to alter critical elements of the pathophysiology of sepsis. Diabetic adults have an increased susceptibility to infection and risk of dying from an infectious disease. Inflammatory and coagulant pathways that are activated during sepsis are also noted to be concurrent in diabetic adults without infection. Clinicians believe urinary tract infections to be more common among adults with diabetes and more severe cases leading to pyelonephritis and bacteremia (Kefteridis, Papadimitraki, Mantadakis, Maraki, Papadakis, Tzifa, \& Samonis, 2009).

A retrospective chart review was conducted by Kefteridis, et al. (2009) at a university hospital. Participants consisted of 88 adults aged 65 and older with diabetes and 118 adults without diabetes. The researchers reviewed charts from January 1997 
through December 2005 of adults hospitalized for acute pyelonephritis. Demographic, clinical and microbiological data was collected from the medical records. Of the study subjects, $27(30.7 \%)$ diabetic and $13(11.0 \%)$ non-diabetic adults developed bacteremia $(\mathrm{p}=0.001)$. The researchers found that subjects with diabetes had a fever for longer periods of time ( 4.5 versus 2.5 days; $\mathrm{p}<0.001)$, longer hospitalization (10 days versus 7 days; $\mathrm{p}<0.001)$, and greater mortality rates $(12.5 \%$ versus $2.5 \% ; \mathrm{p}<0.01)$ than those without diabetes. Limitations of the study include different treatment from physicians over the course of nine years, lack of data regarding DM treatment, and the lack of prior antimicrobial consumption data (Kefteridis et al., 2009).

A retrospective chart review by Yang, Salam, Ong, \& Yang (2011) was completed to compare the organ dysfunction between diabetic and nondiabetic adults. The study reviewed 9,221 charts from Singapore General Hospital in the years 2004 to 2008, 2943 (31.9\%) being diabetic adults. Study participants were greater than 18 years or older. The collected data included demographic information such as ethnicity, age, marital status, gender, dates of admission and discharge, dates of ICU admission and discharge, up to 10 ICD-9 codes, discharge status, and disposition at discharge. The most common organ dysfunction in septic adults were renal (31.5\%), cardiovascular (19.2\%), and respiratory $(10.9 \%)$. Among these septic adults, respiratory dysfunction was less likely in diabetic adults $(9.4 \%)$ than in nondiabetic adults $(11.6 \% ; \mathrm{p}=0.002)$. Renal dysfunction was more likely in diabetic adults than nondiabetic (46.5\% versus $24.4 \%$; $\mathrm{p}<$ 0.001). The diabetic adults in the study had equal hospital lengths of stay, 12.1 days compared to 12.2 days and mortality rates $19.2 \%$ vs $20.0 \%$ in non-diabetic adults. The researchers found protective effects of diabetic adults with good glycemic control before developing sepsis. Limitations of the study include coding errors of data and omission of important diagnoses and complications, conditions that could have affect results. Other limitations include large population over multiple years (Yang et al, 2011). 
Stegenga et al. (2010) conducted a retrospective analysis on adults admitted with sepsis involving 837 ICU adults in 164 different centers. The purpose of the study was to determine if the presence of diabetes adversely affects the outcome of sepsis. The researchers divided adults with suspected infection into two groups: diabetic and nondiabetic. The subjects had to have three or more signs of systemic inflammation. Mortality was assessed after 28 and 90 days and, the infectious agent, markers for coagulation and fibrinolysis were measured throughout the study. In total $22.7 \%$ of septic adults were diabetic. Throughout the length of stay in the hospital, diabetic adults had higher glucose levels. At 28 days mortality rates were equal for diabetic adults and nondiabetic adults (31.4\% and 30.5\%). Although upon admission diabetic adults had slightly higher markers of coagulation, fibrinolysis, and inflammation, and they were generally equal between diabetic and nondiabetic adults during hospitalization. Subjects without diabetes with admission hyperglycemia were associated with higher mortality rates than subjects without admission hyperglycemia (43.0\% versus $27.2 \%$ ). A limitation of study was that the two cohorts were not totally equal in age, APACHE II score, and organ dysfunction at baseline. Discrimination between stress hyperglycemia and undiagnosed diabetes in nondiabetic group, HbA1c would have been valuable. No data was available on type of diabetes, nor any documentation on diabetes complications (Stegenga et al, 2010).

In conclusion, further analysis of the correlation between hyperglycemia and sepsis is needed. The majority of the studies demonstrate that the strict control of glucose decreased the morbidity and mortality of critically ill adults. However, insulin therapy carries an increased risk of hypoglycemia which can be more detrimental then hyperglycemia in some adults. The majority of the studies also discussed protective measures of insulin therapy with diabetic adults and indicated a greater need to investigate the effects of hyperglycemia within the septic non-diabetic adult. Lastly nondiabetic adults need to be closely monitored post-infection for the increased risk of 
developing diabetes type II (Gornik et al., 2010). There is a continued need to study the effects of hyperglycemia within the septic adult and the concurrent effects on lactate levels. 


\section{Theoretical Framework}

The theory of General Adaptation Syndrome (GAS), a borrowed theory from behavioral sciences and physics was the theoretical framework used to guide the research study. Hans Selye, a Hungarian endocrinologist, first used the term 'stress' to describe a biological reaction to homeostasis while researching the effects of hormones on rats. Selye pioneered research on stress and developed the GAS theory in 1956. Selye theorized that stress is a major cause of disease and chronic stress causes long-term chemical adaptations. Stressors can greatly influence normal metabolism leaving a limited supply of adaptive energy (Selye, 1950).

Based on physiology and psychobiology, the stress model can be explained in three stages of the general adaptation syndrome: alarm, resistance and exhaustion. In the first stage the body reacts with "fight or flight" response with the activation of the sympathetic nervous system upon encountering a stressor. Hormones such as adrenalin and cortisol are released into the blood stream to help the body fight the stressor (McEwens \& Wills, 2011). During the first phase, most adults compensate and maintain homeostasis, thus making identifying sepsis difficult. The first stage may be identified as blood glucose levels start to rise, while the adult remains at an increased level of alertness and anxiety begins.

In the second stage, the body responds and compensates with the parasympathetic nervous system. As the body attempts to return physiological function to normal, the blood glucose levels remain high. Cortisol and adrenalin continue to circulate through the body at elevated levels causing increased heart rate, blood pressure and breathing. The second stage can be identified as adults continue with high levels of glucose (McEwens \& Wills, 2011).

At the end of the second stage, the body will remain "on alert" exhausting biological reserves and becoming susceptible to disease and death in the last stage of the stress response. The last stage occurs when the stressor cannot be neutralized. Due to the 
long duration of the stressor the body becomes overwhelmed by the stressor and can no longer compensate to maintain homeostasis. The body becomes dysfunctional and can display a multitude of psychopathologic symptoms such as disorganized thinking, change in personality, delusions, hallucinations, stupor, or violence. The last stage can be displayed when the adult is at its highest stress level and notably at an unstable hemodynamic level as evidence by glucose and lactate levels (McEwens \& Wills, 2011). Response to stressors can be predicted from the three phases within the General Adaptation Syndrome. The theory supports the study by explaining how the stress of infection will impact the glucose levels. Additionally, Selye (1950) observed that even when one's body wants to control or reduce the stress, the ability is limited. The supply of the body's energy is limited in adapting to the stressful environment and is even more compromised when the body is continuously exposed to the stressor. The theory gives explanation and guidance in predicting an adult's response in a stressful event such as IS. Knowing the predicted responses Selye has identified in GAS, combined with the findings of this research, changes in the current sepsis protocol related to optimal glucose management in IS maybe well supported (McEwens \& Wills, 2011). 


\section{Method}

\section{Purpose}

The purpose of the research study was to identify if a relationship exists between hyperglycemia, lactatemia, length of hospital stay and mortality in adults with preexisting DM and IS. The relationship was identified by comparing the glucose and lactic acid levels within the first 48 hours of the hospitalization of the septic event with hospital length of stay and mortality rates. The researcher proposed that individuals with higher glucose levels would have an increase in lactic acid levels resulting in longer lengths of stay and higher mortality rates than those who have a tighter glucose control as defined by a glucose level between 80 and $180 \mathrm{mg} / \mathrm{dl}$.

\section{Research Question}

The research question was: What is the relationship between hyperglycemia and hyperlactatemia on hospital length of stay and mortality based on preexisting diabetes in adults admitted to the emergency department with infectious sepsis?

\section{Design}

The project was a quantitative descriptive study done through a retrospective chart review. Group A included subjects with no existing diagnosis of DM who met the inclusion criteria, to which a collection of lab values, length of stay and mortality data was collected. Group B included subjects with existing diagnosis of DM who met the inclusion criteria, to which a collection of lab values, length of stay and mortality data was collected. The data from both groups were compared and evaluated to distinguish if a relationship existed between elevated glucose levels and lactic acid levels, length of stay and mortality rates.

Site

The data collection took place at Charlton Memorial Hospital (CMH) in Fall River, Massachusetts. The hospital is a nonprofit, non-teaching community hospital that 
is licensed for 285 beds, of which 25 are critical care. Currently CMH evaluates and treats approximately 500 septic patients per year.

\section{Sample}

The sample included adults between 18 and 90 years of age, admitted with the diagnosis of sepsis, recruited from a data base of adults with ICD codes of sepsis with and without previous diagnosis of diabetes. Excluded were pregnant patients, prisoners, and adults with more than five comorbid conditions. The number of charts reviewed were 146 to obtain two sample groups of 25 . Group A included adults diagnosed with diabetes and Group B included non-diabetics adults with sepsis.

\section{Measurement}

A data collection instrument (Appendix A) designed by the student researcher included the following data points: age, serum glucose and serum lactate, diabetes diagnosis, insulin therapy (none, IV infusion, sliding scale), length of stay (LOS) and evidence of hospital mortality. De-identified data collected was entered into an Excel spreadsheet and kept in a locked locker which only the researcher and facility advisor had access to and confidentiality was maintained.

\section{Ethical Considerations}

Ethical considerations for the research study included patient confidentiality. Adults were identified as numbers 1-25 in Group A and Group B, for a total of 50 adults. All recorded information was secured in a confidential folder located in a locked locker. There was no discrimination for race, age or gender. Institutional review board (IRB) approval was obtained from Rhode Island College and was used according to Southcoast Health System Federal Wide Assurance (FWA).

\section{Procedure}

CMH leadership support was obtained from the Assistant Chief Nursing Officer in the form of a written letter (Appendix C). Hospital Information Technology division was contacted for access to EMR for systematic chart reviewing in order to 
locate and approve charts coded by ICD 9 code 995.91 for sepsis. IRB approval was obtained from Rhode Island College and was used according to Southcoast Health System FWA. The chart reviews took place in CMH library. The data retrieved was stored in a folder labeled 'confidential' and kept in a locked locker in the nurse's locker room. De-identified data was recorded into an excel spread sheets (Appendix A and Appendix B). The raw data was destroyed after completion of research study. Confidentiality of all data was maintained throughout research study. Data was accessed by the student researcher only. A total of 146 charts were reviewed between the dates of February 11, 2016 and February 25, 2016. The dates of the charts collected are between January 2015 and December 2015. After all data was collected, results were evaluated. The research was presented to RIC School of Nursing in May, 2016. Results were also shared with the education council and policy review board at Charlton Memorial Hospital for review and revision of sepsis protocol.

\section{Data Analysis}

Initial demographic and study variables were analyzed with descriptive statistics. Quantitative data was obtained for analysis to identify if any relationship exists between elevated glucose and lactic acid levels within the first 48 hours of septic illness, the length of stay and mortality rates of diabetic and non-diabetic patients with a septic illness. A range and mean age was calculated. Mean and standard deviation was calculated for length of hospital stay and mortality rates for both groups and compared. Data collected was presented in a table format.

\section{Enabling Factors and Barriers}

Enabling factors included the support and guidance from Rhode Island College Faculty and Southcoast Hospital Groups Chief Nursing Officer. Limitations to the study were based on availability of charts with inclusion criteria, time of blood draws and if glucose and lactate levels are drawn concurrently. Other barriers included changing of 
electronic medical records from Meditech software to Epic in October 2015, which created a barrier to readily obtaining previous medical charts.

\section{Timeline}

Proposal to CMH was submitted November 10, 2015 and the RIC IRB November $25^{\text {th }}, 2015$. Timeline of the research was from December 1, 2015 to February 28, 2016. Completion of report and findings by April 30, 2016. Presentation of project May 3, 2016.

\section{Desired Outcomes}

The desired outcomes of the research study were to identify if a relationship exists between hyperglycemia, hyperlactatemia, identifying severity of sepsis, hospital length of stay and mortality rate. The researcher will share the findings to support the implementation of glucose management to the existing sepsis protocol at Southcoast Hospital Groups to be current with national standards and Surviving Sepsis Campaign.

\section{Dissemination}

The study findings were presented in May 3, 2016 at the Research Symposium held at Rhode Island College. The final report is accessible through the Electronic Digital Comments of Rhode Island College Library, with additional possible submission to the Critical Care Medicine Journal. Results of the research will be shared with CMH and may impact the sepsis protocol by implementing insulin administration into the sepsis protocol currently inplace. 


\section{Results}

A total of 148 charts were reviewed; 56 charts were excluded because they did not meet the inclusion criteria. Another 42 were eliminated as the patients had developed IS after admission to the hospital as a secondary diagnosis. Thus, the remaining charts $(n=50)$ were reviewed to identify if a relationship occurred between hyperglycemia and hyperlactatemia.

The age range of the adults were from 32 to 87 with a mean of 58.5 years. Group A, adults with preexisting diabetes presenting with IS included 50 percent $(n=25)$ of the adults reviewed. The remaining 50 percent $(n=25)$ were placed in group B which were adults without a preexisting diagnosis of diabetes.

Comparison of the three glucose mean levels, range, and standard deviation are listed in Table 1. The majority of the adults in group A were type II diabetics, however 44 percent $(n=11)$ were maintained on a sliding insulin scale for glycemic control. The remaining 56 percent $(n=14)$ were maintained on oral glycemic agents. The adults in group B had no glycemic control throughout their hospital stay. The first glucose level was drawn on arrival to the emergency department, the second was drawn six hours later and the last glucose was drawn 48 hours after the initial level was drawn, all on the same time frame as the lactate levels.

Table 1

Serial serum glucose levels

\begin{tabular}{|c|c|c|c|c|c|c|c|c|c|}
\hline Group & Mean 1 & Range 1 & SD 1 & Mean 2 & Range 2 & SD 2 & Mean 3 & Range 3 & SD 3 \\
\hline A & 183.2 & $73-436$ & 86.875 & 191.92 & $84-546$ & 92.991 & 127 & $75-283$ & 48.019 \\
\hline B & 122.56 & $85-183$ & 23.024 & 135.36 & $78-192$ & 27.152 & 99.2 & $72-229$ & 29.839 \\
\hline
\end{tabular}

Comparison of the three lactate mean levels, range of lactate levels, and standard deviation are listed in Table 2. All adults were started on the sepsis protocol in the emergency department and administered weight based intravenous fluid administration in accordance to the protocol. The first lactate level was drawn on arrival to the emergency 
department, the second was drawn six hours later and the final lactate level was drawn 48 hours from the first.

Table 2

Serial serum lactate levels

\begin{tabular}{|c|c|c|c|c|c|c|c|c|c|}
\hline Group & Mean 1 & Range 1 & SD 1 & Mean 2 & Range 2 & SD 2 & Mean 3 & Range 3 & SD 3 \\
\hline A & 2.684 & $1.5-5.5$ & 0.733 & 2.192 & $1.2-3.3$ & 0.625 & 1.384 & $0.8-2.6$ & 0.495 \\
\hline B & 3.804 & $1.1-94$ & 2.44 & 3.36 & $1.6-9.7$ & 2.037 & 1.68 & $0.8-3.8$ & 0.726 \\
\hline
\end{tabular}

Figure 1 compares the mean glucose levels to the mean lactate levels in Group A. Figure 2 compares the mean glucose levels to the mean lactate levels in Group B. Group A had higher glucose levels when compared to Group B, however Group B had higher lactate levels. There was a rise in the glucose level taken at the six-hour mark compared to the initial glucose level in both groups.

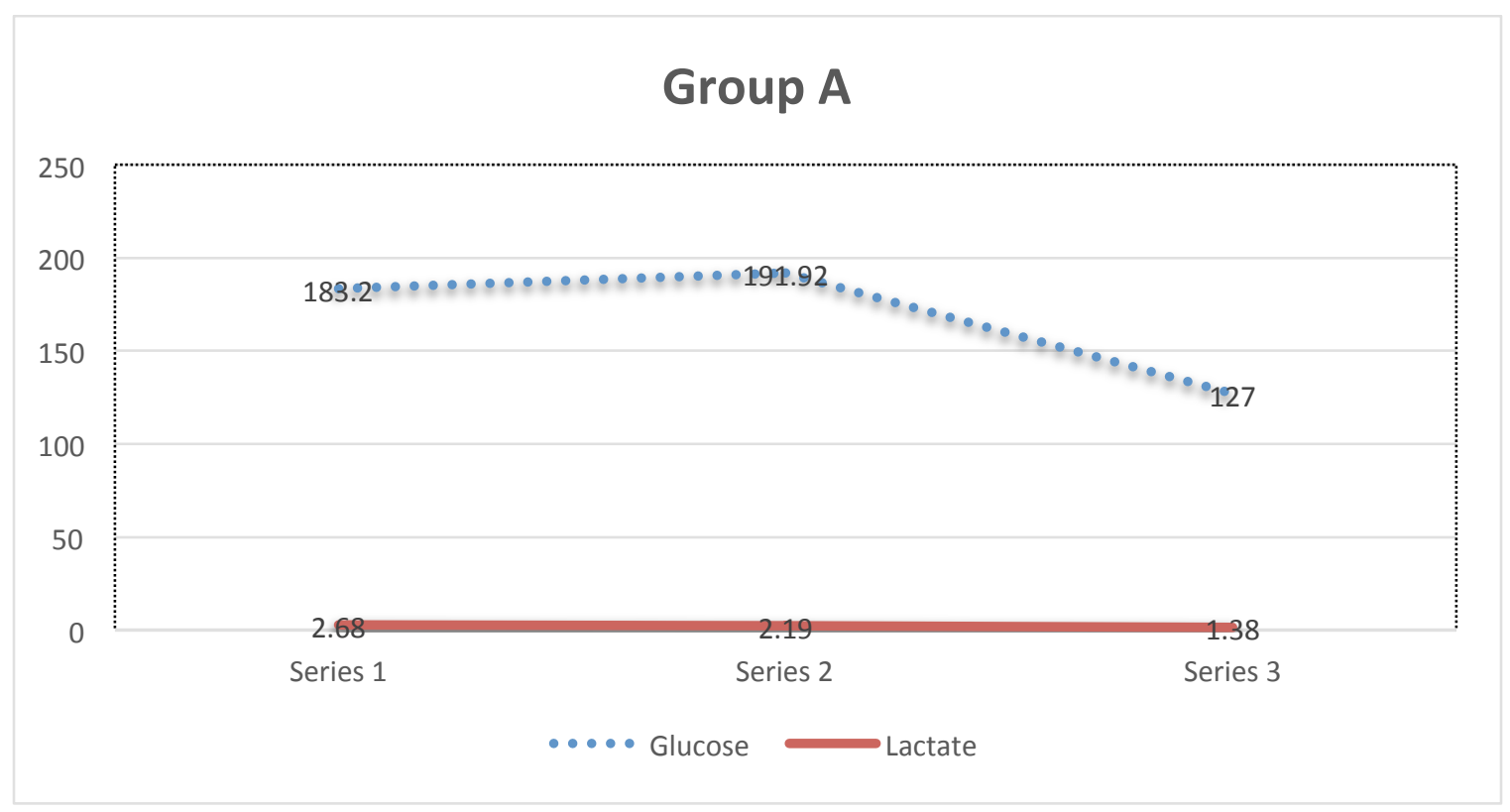

Figure. 1. Serum glucose vs serum lactate levels in Group A. 


\section{Group B}

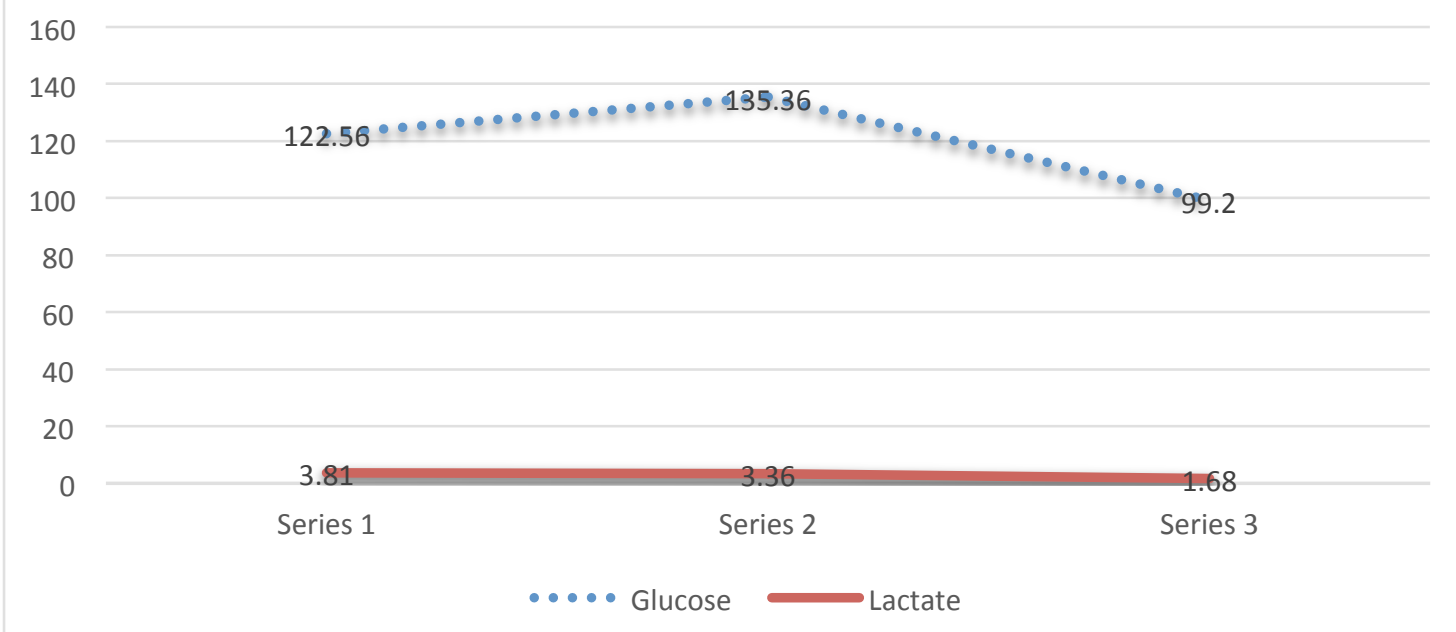

Figure. 2. Serum glucose levels vs serum lactate levels in Group B.

Comparison of the mean length of stay in days, range of length of stay, and percentage of mortality rates are listed in table 3. Group A had no mortalities and a short length in stay when compared to Group B. There was a 12.5 percent $(n=2)$ mortality rate in Group B and a higher in length of stay.

Table 3

Length of stay vs. mortality rates

\begin{tabular}{|c|c|c|c|}
\hline Group & Mean LOS & LOS Range & $\begin{array}{c}\text { Percentage of } \\
\text { Morality }\end{array}$ \\
\hline A & 8.28 & $5-17$ & 0 \\
\hline B & 9.32 & $3-26$ & 12.5 \\
\hline
\end{tabular}




\section{Summary and Conclusions}

Early identification and intervention is the gold standard for treatment of IS in adults presenting to the emergency department (CDC, 2016). IS continues to present in a complex manner making it difficult to identify and diagnosis. Septicemia is currently the sixth most common diagnosis requiring hospitalization in America (CDC, 2016). Between the years 2000 and 2008 the number of adults identified with sepsis doubled, reaching over one million Americas affected each year. Of the adults diagnosed with IS, one half of them will die from the infection. In 2009, sepsis was one of the most expensive hospitalizations, costing nearly 15.4 billion dollars (CDC, 2016).

As it is common to have stress-induced hyperglycemia during critical illness, the

level in which the hyperglycemia becomes harmful is $180 \mathrm{mg} / \mathrm{dl}$ or greater. The Surviving Sepsis Campaign continues to recommend maintaining a serum glucose below $180 \mathrm{mg} / \mathrm{dl}$ in all septic patients with and without diabetes (Surviving Sepsis Campaign, 2016). Although the risk for diabetic patients to become septic is greater than a nondiabetic adult, the effects of hyperglycemia are equally as harmful. The effects of hyperglycemia on critically ill adults continues to be a leading cause of mortality in septic adults, thus supporting the recommendations of glycemic control (CDC, 2016).

The purpose of the study was to identify if a relationship exists between hyperglycemia, hyperlactatemia, hospital length of stay and mortality rates in adults with IS. It was proposed that adults with higher glucose levels would have higher lactate levels therefore increasing length of hospital stay and higher risk of mortality. The study was guided by the General Adaptation Theory (Salye, 1950). A retrospective chart review was conducted; 148 charts were reviewed of which 50 met the inclusion criteria. Group A $(n=25)$ was defined as adults with preexisting diabetes. Group B $(n=25)$ was defined as adults without a preexisting diagnosis of diabetes. The data showed that when the two groups were compared, Group A had higher glucose levels then Group B. Although Group B had higher lactate levels and an increase in hospital stay and a 12.5 percent 
mortality rate within the group. Group A had a shorter length of stay with zero mortality within the group.

The researcher's projected outcome was for Group B nondiabetic adults to have higher glucose levels leading to higher lactate levels and increasing the LOS and higher mortality rates. Although the results indicated Group B to have lower glucose levels, they did have higher lactate levels and longer LOS as well as a higher mortality rate. The results suggest Glycemic agents did not decrease glucose and could not determine if they had any protective effect for lowering lactate levels in Group A. The research did not prove a relationship between hyperglycemia and hyperlactate levels resulting in longer LOS and or higher mortality rates. As stated in Schuetz et al. (2011) study, insulin provides more than one protective effect. Insulin not only lowers glucose levels, it has a direct effect on cell function and the physiologic response to infection. Thus suggesting this in the study, although diabetic adults in Group A had higher glucose levels, the subjects in Group A had better overall post sepsis outcomes then nondiabetic patients in Group B, as evidence by shorter LOS and no mortalities within the study.

\section{Limitations}

Limitations to the study include small sample size, availability of charts with inclusion criteria, time of blood draws and if glucose and lactate levels are drawn concurrently. Other limitations included changing of electronic medical records from Meditech software to Epic in October 2015, which created a barrier to readily obtaining previous medical charts.

\section{Ethical/Social Issues}

No ethical issues at this time for the study, however social issues would be for access to medical care and proper glucose control for patients and ability for glycemic control.

\section{Quality Measures}


Quality measures for potential timing and accurate measures for obtaining blood sugar levels. Administration of glycemic agent and nursing competency on following IS protocol based on national standards.

\section{Cost Considerations}

Minimal cost considerations for serum blood draws, increased nursing staff time to interpret protocol and administration of glycemic agent. 


\section{Recommendations and Implications for Advanced Nursing Practice}

Advanced Practice Registered Nurses (APRN) have a responsibility to the critically ill adult to provide the best care according to evidenced based consensus statements by following the Surviving Sepsis Campaign recommendations of close glycemic control in the septic population regardless of preexisting diagnosis of diabetes. Prevention and management of sepsis the critically ill adult is complex and requires accurate identification of the onset of SIRS and diagnosis of IS. Specific to the findings in this study, APRNs can provide the implementation of evidence based protocols that includes use of glycemic control with all adults with IS. Methods for assessing, recording, and documenting a change or identification of vital signs, and evaluation of key laboratory findings must be clearly specified and evidence based.

Evidence based practice drives quality improvement, and benefits both the hospitals and the adults receiving care. Providing care considered to be the gold standard will contribute to a decrease in hospital LOS and decrease rates of IS mortality. Initiating glucose control for all adults with and without diabetes will not only improve the outcomes, but will cut down on long term costs and potential complications from prolonged hyperglycemia.

APRNs continue to advocate for patients and help improve education throughout healthcare facilities and implementing evidenced based practice. Contributing to advancement of policies and procedures related to IS will provide improved care management of patients and help health care systems to reduce cost. APRNs are in an ideal position to educate and become leaders, but also to continue to advocate for improved patient outcomes as part of an interdisciplinary team. Advocating for policy change to keep current with evidence based practice and research is one of the ways in which the APRN can change the trajectory of health and disease management. This study contributes to the evidence that hyperglycemia may negatively impact patient outcome in IS and the APRN has a role in translating current research into optimal patient care. 


\section{References}

Ali, N.A., O'Brien, J.M., Dungan, K., Phillips, G., Marsh, C.B., Lemeshow, S., \& Preiser, J. (2008). Glucose variability and mortality in patients with sepsis. Critical Care Medicine, 36 (8), 2316-2321. doi 10.1097/CCM.0b013e3181810378

American Diabetes Associations (2015, May 18). Statistics about diabetes. Retrieved from: http://www.diabetes.org/diabetes-basics/statistics/?loc=db-slabnav

Centers for Disease Control and Prevention (2013, May 22). Diabetes. Retrieved from: http:www.cdc.gov/sepsis/

Digman, C., Borto, D., \& Nasraway, S. (2005). Hyperglycemia in the critically ill. Nutrition in Clinical Care 8(2), 93-101.

Green, J. P., Berger, T., Garg, N., Horeczko, T., Suarez, A., Radeos, M. S., \& Panacek, E. A. (2012). Hyperlactatemia affects the association of hyperglycemia with mortality in nondiabetic adults with sepsis. Academic Emergency Medicine, 19(11), 1268-1275. doi 10.1111/acem.12015

Gornik, I., Vujaklija, A., Lukic, E., Madzarac, G., \& Gasparovic, V. (2010). Hyperglycemia in sepsis is a risk factor for development of type II diabetes. Journal of Critical Care, 25, 263-269. doi 10.1016/j.jcrc.2009.10.002

Guthrie, R. A., \& Guthrie, D. W. (2004). Pathophysiology of diabetes mellitus. Journal of Critical Care, 27(2), 113-125.

Johnson, J. A., \& Horn, E. R. (2011). The effects of correction insulin and basal insulin on inpatient glycemic control. Journal of Medsurg Nursing, 20(4), 187-193.

Kaufman, S. (2008). Normalization of glucose in the intensive care unit: Does one size really fit all? Critical Care Medicine, 36(8), 2448-2449. doi 10.1097/CCM.0b013e318181129a

Kofteridis, D. P., Papadimitraki, E., Mantadakis, E., Maraki, S., Papadakis, J. A., Tzifa, G., \& Samonis, G. (2009). Effects of diabetes mellitus on the clinical and microbiological features of hospitalized elderly patients with acute pyelonephritis. 
The American Geriatrics Society, 57, 2125-2128. doi 10.111/j.1532-

$5415.2009 .02550 . x$

Lin, J. N., Tsai, Y. S., Lai, C. H., Chen, Y. H., Tsai, S. S., Lin, H. L., Huang, C. K., \&

Lin, H. H. (2009). Risk factors for mortality of bacteremic patients in the emergency department. Society for Academic Emergency Medicine, 16(8), 749755. doi 10.1111/j.1553-2712.2009.00468.x

Lorencio, C., Leal, Y., Bonet, A., Bondia, J., Palerm, C.C., Tache, A., \& Vehi, J. (2012). Real - time continuous glucose monitoring in an intensive care unit: Better accuracy in patients with septic shock. Diabetes Technology \& Therapeutics, 14(7), 568-575. doi 10.1089/dia.2012.0008

Mayo Clinic (2014, July). Sepsis. Retrieved from http://www.mayoclinic.org/diseasesconditions/sepsis/basics/definition/con-20031900

McEwen, M., \& Wills, E. M. (2011). Theoretical Basis for Nursing ( $3^{\text {rd }}$ ed.). Philadelphia, PA: Lippincott Williams \& Wilkins.

Michaels, R., K. \& Dorman, T. (2004). Evidence based medicine in the intensive care unit: Improving care for the septic patient. Department of Anesthesiology and Critical Care Medicine, John Hopkins University, 24, 41-49. doi 10.1053/j.sane.2004.11.009

Rhee, C., Murphy, M. V., Li, L., Platt, R., \& Klompas, M. (2015). Lactate testing in suspected sepsis: trends and predictors of failure to measure levels. Society of Critical Care Medicine and Wolters Kluwer Health, Inc., 43(8), 1669-1676. doi 10.1097/CCM.0000000000001087

Revelly, J. P., Tappy, L., Martinez, A., Bollmann, M., Cayeux, M. C., Berger, M. M., \& Chiolero, R. L. (2005). Lactate and glucose metabolism in severe sepsis and cardiogenic shock. Society of Critical Care Medicine and Lippicott Williams \& Wilkins, 33(10), 2235-2240. doi 10.1097/01.CCM.0000181525.99295.8F 
Schuetz, P., Castro, P., \& Shapiro, N. (2011). Diabetes and sepsis: Preclinical findings and clinical relevance. Diabetes Care, 34,771-778. doi 10.2337/dc10-1185

Schuetz, P., Jones, A., Howell, M., Trzeciak, S., Ngo, L., Younger, J., \& Shapiro, N.I., (2011, November). Diabetes is not associated with increased mortality in emergency department patients with sepsis. Annals of Emergency Medicine, 58(5), 438-444. doi 10.1016/j.annemergmed.2011.03.052

Selye, H. (1950). Stress and the general adaptation syndrome. British Medical Journal, 1383-1392. Retrieved from: http://www.ncbi.nlm.nih.gov/pmc/articles/PMC2038162/pdf/brmedj036030003.pdf

Stegenga, M.E., Vincent, J., Vail, G.M., Xie, J., Haney, D.J., Williams, M., \& Van der Poll, T. (2010). Diabetes does not alter mortality or hemostatic and inflammatory responses in patients with severe sepsis. Critical Care Medicine, 38(2), 539-545. doi 10.1097/CCM.0b013e3181c02726

Spapen, H. D. (2008). The glycemia threat in sepsis: Too high, too low, or too ... variable!. Critical Care Medicine, 36, 2459-2460. doi 10.1097/CCM.0b013e3181811260

Surviving Sepsis Campaign (2015, July). Retrieved from http://www.sccm.org/Communications/CriticalConnections/Archives/Pages/SCCM-Unveils-Insulin-Infusion-Guidelines-forGlycemic-Control.aspx

Thompson, T. (2008). Glucose control in sepsis. Clinics in Chest Medicine, Elsevier, 29, 713-720. doi 10.1016/j.ccm.2008.06.002

Van den Berghe, G. (2003). Insulin therapy for critically ill patient. Clinical Cornerstone Complications of Diabetes, 5(2), 56-63. 
Yang, K. S., Salam, Z.A., Ong, B.C., \& Yang, K.S. (2011). Respiratory dysfunction in patients with sepsis: Protective effect of diabetes mellitus. American Journal of Critical care, 20, e41-e47. 10.4037/ajcc2011391 


\section{Appendix A}

\begin{tabular}{|c|c|c|c|c|c|c|c|c|c|}
\hline Group A & Glucose 1 & Glucose 2 & Glucose 3 & Lactate 1 & Lactate 2 & Lactate 3 & Age & LOS & Mortality \\
\hline 1 & & & & & & & & & \\
\hline 2 & & & & & & & & & \\
\hline 3 & & & & & & & & & \\
\hline 4 & & & & & & & & & \\
\hline 5 & & & & & & & & & \\
\hline 6 & & & & & & & & & \\
\hline 7 & & & & & & & & & \\
\hline 8 & & & & & & & & & \\
\hline 9 & & & & & & & & & \\
\hline 10 & & & & & & & & & \\
\hline 11 & & & & & & & & & \\
\hline 12 & & & & & & & & & \\
\hline 13 & & & & & & & & & \\
\hline 14 & & & & & & & & & \\
\hline 15 & & & & & & & & & \\
\hline 16 & & & & & & & & & \\
\hline 17 & & & & & & & & & \\
\hline 18 & & & & & & & & & \\
\hline 19 & & & & & & & & & \\
\hline 20 & & & & & & & & & \\
\hline 21 & & & & & & & & & \\
\hline 22 & & & & & & & & & \\
\hline 23 & & & & & & & & & \\
\hline 24 & & & & & & & & & \\
\hline 25 & & & & & & & & & \\
\hline
\end{tabular}




\section{Appendix B}

\begin{tabular}{|c|c|c|c|c|c|c|c|c|c|}
\hline Group B & Glucose 1 & Glucose 2 & Glucose 3 & Lactate 1 & Lactate 2 & Lactate 3 & Age & LOS & Mortality \\
\hline 1 & & & & & & & & & \\
\hline 2 & & & & & & & & & \\
\hline 3 & & & & & & & & & \\
\hline 4 & & & & & & & & & \\
\hline 5 & & & & & & & & & \\
\hline 6 & & & & & & & & & \\
\hline 7 & & & & & & & & & \\
\hline 8 & & & & & & & & & \\
\hline 9 & & & & & & & & & \\
\hline 10 & & & & & & & & & \\
\hline 11 & & & & & & & & & \\
\hline 12 & & & & & & & & & \\
\hline 13 & & & & & & & & & \\
\hline 14 & & & & & & & & & \\
\hline 15 & & & & & & & & & \\
\hline 16 & & & & & & & & & \\
\hline 17 & & & & & & & & & \\
\hline 18 & & & & & & & & & \\
\hline 19 & & & & & & & & & \\
\hline 20 & & & & & & & & & \\
\hline 21 & & & & & & & & & \\
\hline 22 & & & & & & & & & \\
\hline 23 & & & & & & & & & \\
\hline 24 & & & & & & & & & \\
\hline 25 & & & & & & & & & \\
\hline
\end{tabular}


Appendix C

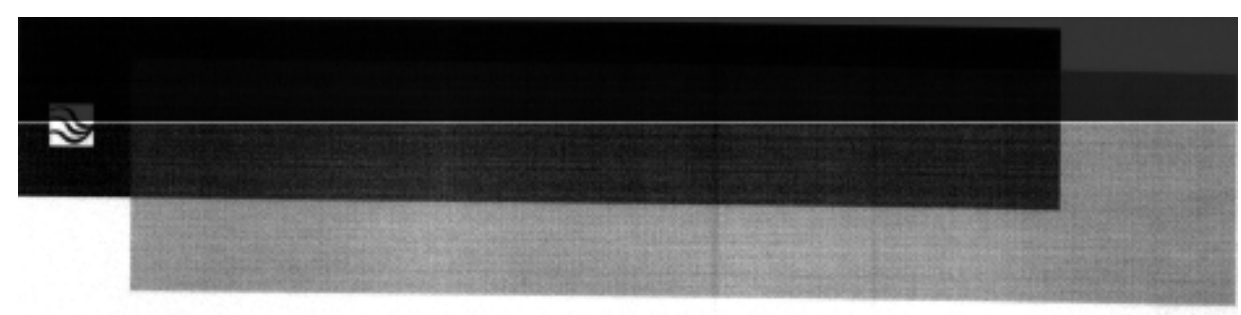

January 10,2016

To whom it mary concern

This is a letter of support for Katie Lineman, RN Rhode Island College student. She will be partnering with the Chartton Memorial Hospital Emergency Department. As the Associate Chief Nursing Officer, I support this project by Ms. Lineman. However, prior to beginning working with subjects this project Ms. Lineman must receive a letter of endorsement from The Clinical Trials office at Southcoast Health and Institutional Review Board (IRB) approval. Ms. Lineman must use a designated IRB on the Southcoast Health Federalwide Assurance (FWA) for her review of the study.

Support contingencies:

- Southcoast Health Clinical Trials endorsement

- Southcoast Health Clinical Trials letter provided to investigator

- Institutional Review Board approval

- IRB must be on the Southcoast Health FWA

Southcoast Health is proud to partner with Ms. Lineman and Rhode Island College. Thank you for reaching out to us for this project. I personally look forward to reviewing your findings.

Regards,

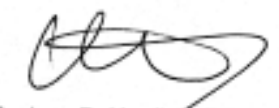

Andrew D. Harcling, RN

Associate Chief Nursing Officer

Chariton Memorial Hospital

s Southcoast Health 